\title{
Estimación de la prevalencia del trastorno por déficit de atención con o sin hiperactividad (TDAH) en población escolar de la Comunidad Autónoma de Canarias
}

\author{
Juan E. Jiménez ${ }^{1}$, Cristina Rodríguez ${ }^{1}$, Juan Camacho ${ }^{1}$, \\ Miguel Afonso ${ }^{2}$ y Ceferino Artiles ${ }^{2}$ \\ ${ }^{1}$ Universidad de La Laguna, ${ }^{2}$ Consejería de Educación, Universidades y Sostenibilidad \\ del Gobierno de Canarias
}

\begin{abstract}
Este estudio ha tenido por finalidad determinar la prevalencia del Trastorno por Déficit de Atención con o sin Hiperactividad (TDAH) en la Comunidad Autónoma de Canarias, en la población de 6 a 12 años. Para ello, el muestreo utilizado fue polietápico estratificado y proporcional por conglomerados. Los estratos fueron el tipo de colegio, la Isla y el nivel educativo, y los conglomerados fueron los colegios. A partir de una muestra de estudio de 118.467 alumnos escolarizados en centros ordinarios, se obtuvo una muestra al azar de 2.395 alumnos. Se utilizaron las escalas Attention-Deficit/Hiperactivity Disorder Rating Scales IV (ADHD RS-IV) modificadas para padres y maestros. Se encontró una tasa global de prevalencia del $4.9 \%$, de los cuales el $3.1 \%$ fueron del subtipo inatento, el $1.1 \%$ hiperactivo, y el $0.7 \%$ combinado. Solo se encontraron diferencias significativas en función del sexo, donde la incidencia fue superior en varones. Las implicaciones educativas y socio sanitarias de los hallazgos encontrados nos sugieren la importancia de llevar a cabo una identificación, diagnóstico y tratamiento temprano, lo que ayudará a que los niños identificados con TDAH puedan desarrollar todo su potencial, y con ello una reducción importante del gasto público.
\end{abstract}

Palabras clave: Prevalencia, TDAH, DSM-IV-R, Educación Primaria, Islas Canarias.

Estimating the prevalence of attention deficit disorder with or without hyperactivity $(A D H D)$ in school children of the Canary Islands. The main purpose of this study has been to determine the prevalence of Attention Deficit Disorder with or without Hyperactivity (ADHD) in the Canary Islands in the population aged 6 to 12 years old. For this, the sampling design used was multistage stratified cluster proportional. The strata were the type of school, the Island and educational level, and conglomerates were the schools. From a study sample of 118.467 students enrolled in mainstream schools, we obtained a sample of 2.395 students. Scales were used Attention-Deficit/Hiperactivity Disorder Rating Scales IV (ADHD RS-IV) for parents and teachers. We found an overall prevalence of $4.9 \%$, of which $3.1 \%$ were inattentive subtype, $1.1 \%$ hyperactive, and $0.7 \%$ combined. The prevalence rate was different depending on sex differences; the incidence was higher in males. The educational implications and social health of our findings suggest the importance of carrying out an identification, diagnosis and early treatment that will help children with ADHD can develop their full potential, and thus a significant reduction in public spending.

Key words: Prevalence, ADHD, ADHD RS-IV, elementary grades, The Canary Islands.

Correspondencia: Juan E. Jiménez. Departamento de Psicología Evolutiva y de la Educación. Universidad de La Laguna. Campus de Guajara, C.P. 38200. Islas Canarias (España). E-mail: ejimenez@ull.es 
El Trastorno por déficit de atención con o sin hiperactividad (TDAH) ha sido identificado por la Organización Mundial de la Salud (OMS) como inquietud prioritaria sobre la base de su prevalencia o su potencial, de causar minusvalías, de sus posibilidades terapéuticas de recuperación, especialmente a nivel de atención primaria de la salud, y de sus consecuencias a largo plazo. El CIE-10 de la OMS, 1992, criterios diagnósticos del TDAH, hace mención a los trastornos hipercinéticos, siendo los rasgos principales el déficit de atención y la hiperactividad, los que se deben manifestar en más de una situación, y que producen relaciones sociales desinhibidas, comportamiento antisocial y baja autoestima. Se trata de un trastorno neurobiológico que se manifiesta en dificultades de autorregulación en tres aspectos concretos: la capacidad para mantener la atención; el control de la impulsividad y el grado de actividad. El déficit de atención se manifiesta en que el niño se aburre enseguida y se distrae con facilidad. Otra característica típica del TDAH es la impulsividad, los niños con TDAH son impacientes, interrumpen en ocasiones en las que no es adecuado, les cuesta pararse y pensar en las consecuencias de sus acciones, y no tienden a planificar sus actos futuros. El tercer rasgo distintivo es la hiperactividad. Los niños con TDAH parecen incansables y nunca se están quietos. Les cuesta mucho estar sentados, y cuando lo consiguen no dejan de mover las piernas, tocar todo lo que está a su alcance, dar golpecitos con los dedos y con los pies. Todos estos síntomas suelen provocar malestar a su alrededor, especialmente entre aquellos adultos que consideran erróneamente que se trata de una manifestación de mala educación. Este problema incide en el desarrollo social, escolar y familiar de quienes lo padecen, y no sólo en la etapa escolar, sino también en la vida de quienes en el futuro llegarán a ser adolescentes y posteriormente adultos.

\section{Legislación educativa en la Comunidad Autónoma de Canarias sobre el TDAH}

La Ley Orgánica 2/2006, de 3 de mayo, de Educación, en su artículo 71 recoge que corresponde a las Administraciones educativas asegurar los recursos necesarios para que los alumnos y alumnas que requieran una atención educativa diferente a la ordinaria, por presentar necesidades específicas de apoyo educativo (NEAE), puedan alcanzar el máximo desarrollo de sus capacidades personales. Según lo dispuesto en el artículo 10 del Decreto 104/2010, de 29 de julio (BOC n ${ }^{\circ}$ 154, de 6 de agosto), en el ámbito de la enseñanza no universitaria de Canarias, la Administración educativa establecerá las coordinaciones y acuerdos que procedan con otras Administraciones, especialmente las competentes en materia sanitaria y de bienestar social, para facilitar la detección temprana y favorecer actuaciones preventivas desde los primeros ciclos de la escolaridad.

En este mismo Decreto se establece que "Un alumno o alumna presenta necesidades específicas de apoyo educativo por un trastorno por déficit de atención con o sin hiperactividad cuando su conducta manifiesta un patrón persistente de desatención 
y/o hiperactividad-impulsividad que es más frecuente y grave que el observado en escolares de su edad, repercutiendo negativamente en su vida social, escolar y familiar. Estos síntomas debe estar presentes tanto en el ámbito escolar como familiar y alguno de ellos haber aparecido antes de los siete años, no debiendo estar motivados por otro tipo de trastornos claramente definidos".

En el artículo 10, numeral 3, de la ya citada Ley Orgánica 2/2006, de 3 de mayo, de Educación, se enfatiza la identificación y evaluación lo antes posible del alumnado con NEAE por los equipos de orientación educativa y psicopedagógicos dependientes de la Consejería de Educación, además del fomento de planes de actuación adecuados a dichas necesidades en coordinación con los servicios socio-sanitarios de la zona. Y son, por ello, estas entidades las que deben fomentar evaluaciones cada vez más tempranas y efectivas que hagan que las NEAE derivadas de TDAH sean detectadas lo antes posible. Sin embargo, para nuestro conocimiento no disponemos aún de instrumentos diseñados con esta finalidad para nuestro contexto cultural y educativo normativizados en la población escolar canaria. De esta manera, el disponer de tales instrumentos, permitirá detectar de forma temprana aquellos niños con riesgo de presentar en el futuro dificultades de aprendizaje en lectura.

\section{Estimación de la prevalencia del TDAH}

Han existido intentos por determinar las tasas de prevalencia del TDAH en comunidades escolares (Buitelaar y Van Engeland, 1996; Cardo, Servera y Llobera, 2007; Cardo y Serveraó, 2005; Cornejo et al., 2005; Montiel-Nava, Peña, y MontielBarbero, 2003, Montiel-Nava et al., 2002; Pineda, Lopera, Henao, Palacio y Castellanos, 2001). Cuando se utilizan los criterios del DSM-IV la mayoría de autores estiman la prevalencia global del TDAH entre el 3-7\% y existen márgenes de variabilidad en función de la edad, género, nivel sociocultural, subtipos, etc. En cuanto a la prevalencia de subtipos, hay cierta coincidencia en que el TDA-C registra mayor prevalencia, seguido del predominantemente inatento TDA-I y subtipo hiperactivo impulsivo TDAH. De especial relevancia por sus implicaciones para la investigación sobre la prevalencia del TDAH es el tema de la naturaleza multidimensional de los criterios diagnósticos que definen el TDAH. Ya que, por ejemplo, no hay un único tipo de atención, ni de impulsividad, ni siquiera de sobreactivación motora. Así, se ha establecido la presencia como mínimo de una atención selectiva y una atención sostenida, siendo esta última más típica del TDAH combinado y la primera del TDA-I. Del mismo modo, se ha diferenciado una impulsividad cognitiva (estilo general de aprendizaje y afrontamiento de tareas) y una impulsividad motora (falta de control motor, incapacidad para guardar turno, etc.) siendo esta última más definitoria del TDAH combinado TDA-C, mientras la primera se asocia más a niños que presentan dificultades de aprendizaje. Por otra parte, parece ser que son los varones los más 
afectados en comparación a las mujeres, encontrándose la mayor prevalencia en el rango de edad 6-9 años. Asimismo, se pone de relieve que las grandes diferencias metodológicas entre los estudios dificultan la tarea de revisión.

Para nuestro conocimiento no disponemos de datos epidemiológicos sobre el TDAH en las Islas Canarias. Por ello, el objetivo principal de este estudio ha sido estimar la tasa de prevalencia del TDAH en la Comunidad Autónoma de Canarias a través de la escala ADHD en la población escolar de edades comprendidas entre 6 y 12 años. Asimismo, se ha pretendido analizar si la distribución de los diferentes subtipos TDAH es diferente según el sexo, isla, tipo de colegio y curso.

\section{MÉTODO}

\section{Participantes}

La población la formaban todos los niños del nivel $1^{\circ}$ a $6^{\circ}$ de Educación Primaria en el curso escolar 2010-2011 perteneciente a todos los centros escolares de las Islas Canarias. En total eran 118.467 escolares, de los cuales 93.650 provenían de colegios públicos y 24.817 de colegios concertados. Cuando se utilizan los criterios del DSM-IV la mayoría de los autores (Buitelaar y Van England, 1996; Cardo y Servera, 2005; Cornejo et al., 2005; Montiel et al., 2002, 2003; Pineda et al., 2001) estiman la prevalencia global del TDAH entre el 3 y el 7\%. Con la media de estos dos valores se tuvo una primera estimación de la prevalencia del TDAH: en el 5\%. Por esta razón la varianza de la proporción dio el valor de 0.0475. Entonces, para un universo de 118.467 escolares, aplicando la corrección del tamaño muestral para poblaciones finitas, con un nivel de confianza del $95 \%$ y un error máximo del $1 \%$ se obtuvo un tamaño muestral de 1.796. Para prever la posible mortandad experimental se elevó el tamaño muestral hasta 2.395. Lo que, en la práctica produjo un error máximo en torno al $0.75 \%$. La distribución de este tamaño por nivel, isla y tipo de colegio aparece en la tabla 1.

\section{Instrumento}

Se utilizó la escala Attention-Deficit/Hiperactivity Disorder Rating Scales IV (ADHD RS-IV) modificada para padres y maestros. Esta escala consta de una subescala de inatención (9 ítems), otra de hiperactividad/impulsividad (9 ítems) y la total (18 ítems). Cada ítem puntúa de 0 a 2 puntos. Se obtuvo un coeficiente de consistencia interna para la escala total de profesores de $.94 \mathrm{y}$ de .90 para padres.

\section{Procedimiento}

La técnica de muestreo utilizado fue polietápico estratificado y proporcional por conglomerados. Los estratos fueron el tipo de colegio (público, concertado), la isla (El Hierro, Fuerteventura, Gran Canaria, La Gomera, La Palma, Lanzarote y Tenerife) y 
el nivel educativo (del $1^{\circ}$ al $6^{\circ}$ nivel). Los conglomerados fueron los colegios. Dentro de cada isla se eligieron al azar un número de colegios (proporcional al tamaño del estrato, es decir, al número de niños por isla). En cada uno de estos colegios, por cada nivel educativo, se eligieron al azar cinco niños de todos los que estaban en ese nivel. De esta manera, la fracción muestreada por nivel educativo varió entre el 10 y el 20\%, ya que en cada nivel había entre 25 y 50 niños aproximadamente. En algunos pocos casos el número de niños elegidos al azar por nivel no fue de cinco, fue de cuatro o seis debido a que había pocos colegios disponibles al cruzar isla y tipo de colegio. Esto se debió a la escasez de colegios en algunos cruces de isla y tipo de colegio.

Tabla 1. Distribución de la muestra de estudio según isla, nivel educativo y tipo de colegio

\begin{tabular}{|c|c|c|c|c|c|c|c|c|c|c|c|}
\hline \multirow[b]{2}{*}{$\begin{array}{c}\text { TIPO } \\
\text { COLE } \\
\text { GIO }\end{array}$} & & & & \multicolumn{7}{|c|}{ ISLA } & \multirow{2}{*}{$\begin{array}{c}\text { Total } \\
\text { CANARIA } \\
\mathrm{S}\end{array}$} \\
\hline & & & & EL HIERRO & $\begin{array}{c}\text { FUERTEVEN } \\
\text { TURA }\end{array}$ & $\begin{array}{c}\text { GRAN } \\
\text { CANARI } \\
\mathrm{A}\end{array}$ & $\begin{array}{c}\text { LA } \\
\text { GOMER } \\
\text { A }\end{array}$ & $\begin{array}{c}\text { LA } \\
\text { PALM } \\
\text { A }\end{array}$ & $\begin{array}{c}\text { LANZAROT } \\
\text { E }\end{array}$ & $\begin{array}{c}\text { TENERIF } \\
\mathrm{E}\end{array}$ & \\
\hline \multirow{14}{*}{ 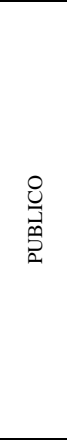 } & \multirow{12}{*}{$\sum_{Z}^{+1}$} & \multirow{2}{*}{$1^{\circ}$} & $\mathrm{N}$ & 10 & 18 & 116 & 15 & 14 & 25 & 98 & 296 \\
\hline & & & $\%$ del total & $.6 \%$ & $1.0 \%$ & $6.5 \%$ & $.8 \%$ & $.8 \%$ & $1.4 \%$ & $5.5 \%$ & $16.5 \%$ \\
\hline & & \multirow{2}{*}{$2^{\circ}$} & $\mathrm{N}$ & 11 & 19 & 120 & 15 & 15 & 26 & 95 & 301 \\
\hline & & & $\%$ del total & $.6 \%$ & $1.1 \%$ & $6.7 \%$ & $.8 \%$ & $.8 \%$ & $1.5 \%$ & $5.3 \%$ & $16.8 \%$ \\
\hline & & \multirow{2}{*}{$3^{\circ}$} & $\mathrm{N}$ & 8 & 18 & 121 & 15 & 15 & 23 & 97 & 297 \\
\hline & & & $\%$ del total & $.4 \%$ & $1.0 \%$ & $6.8 \%$ & $.8 \%$ & $.8 \%$ & $1.3 \%$ & $5.4 \%$ & $16.6 \%$ \\
\hline & & \multirow{2}{*}{$4^{\circ}$} & $\mathrm{N}$ & 11 & 12 & 129 & 15 & 15 & 23 & 97 & 302 \\
\hline & & & $\%$ del total & $.6 \%$ & $.7 \%$ & $7.2 \%$ & $.8 \%$ & $.8 \%$ & $1.3 \%$ & $5.4 \%$ & $16.9 \%$ \\
\hline & & \multirow{2}{*}{$5^{\circ}$} & $\mathrm{N}$ & 10 & 17 & 122 & 15 & 15 & 25 & 97 & 301 \\
\hline & & & $\%$ del total & $.6 \%$ & $1.0 \%$ & $6.8 \%$ & $.8 \%$ & $.8 \%$ & $1.4 \%$ & $5.4 \%$ & $16.8 \%$ \\
\hline & & \multirow{2}{*}{$6^{\circ}$} & $\mathrm{N}$ & 9 & 20 & 118 & 15 & 15 & 24 & 91 & 292 \\
\hline & & & $\%$ del total & $.5 \%$ & $1.1 \%$ & $6.6 \%$ & $.8 \%$ & $.8 \%$ & $1.3 \%$ & $5.1 \%$ & $16.3 \%$ \\
\hline & \multirow{2}{*}{\multicolumn{2}{|c|}{ Total }} & $\mathrm{N}$ & 59 & 104 & 726 & 90 & 89 & 146 & 575 & 1789 \\
\hline & & & $\%$ del total & $3.3 \%$ & $5.8 \%$ & $40.6 \%$ & $5.0 \%$ & $5.0 \%$ & $8.2 \%$ & $32.1 \%$ & $100.0 \%$ \\
\hline \multirow{14}{*}{ 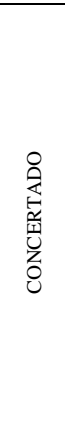 } & \multirow{12}{*}{$\sum_{\text {Z }}^{\overrightarrow{1}}$} & \multirow{2}{*}{$1^{\circ}$} & $\mathrm{N}$ & - & 9 & 35 & - & 12 & 11 & 36 & 103 \\
\hline & & & $\%$ del total & & $1.5 \%$ & $5.8 \%$ & & $2.0 \%$ & $1.8 \%$ & $5.9 \%$ & $17.0 \%$ \\
\hline & & \multirow{2}{*}{$2^{\circ}$} & $\mathrm{N}$ & - & 9 & 35 & - & 11 & 11 & 36 & 102 \\
\hline & & & $\%$ del total & & $1.5 \%$ & $5.8 \%$ & 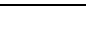 & $1.8 \%$ & $1.8 \%$ & $5.9 \%$ & $16.8 \%$ \\
\hline & & \multirow{2}{*}{$3^{\circ}$} & $\mathrm{N}$ & - & 9 & 34 & - & 11 & 11 & 35 & 100 \\
\hline & & & $\%$ del total & & $1.5 \%$ & $5.6 \%$ & & $1.8 \%$ & $1.8 \%$ & $5.8 \%$ & $16.5 \%$ \\
\hline & & \multirow{2}{*}{$4^{\circ}$} & $\mathrm{N}$ & - & 9 & 35 & - & 12 & 11 & 38 & 105 \\
\hline & & & $\%$ del total & & $1.5 \%$ & $5.8 \%$ & & $2.0 \%$ & $1.8 \%$ & $6.3 \%$ & $17.3 \%$ \\
\hline & & \multirow{2}{*}{$5^{\circ}$} & $\mathrm{N}$ & - & 9 & 35 & - & 11 & 11 & 33 & 99 \\
\hline & & & $\%$ del total & & $1.5 \%$ & $5.8 \%$ & & $1.8 \%$ & $1.8 \%$ & $5.4 \%$ & $16.3 \%$ \\
\hline & & \multirow{2}{*}{$6^{\circ}$} & $\mathrm{N}$ & - & 8 & 34 & - & 12 & 11 & 32 & 97 \\
\hline & & & $\%$ del total & & $1.3 \%$ & $5.6 \%$ & & $2.0 \%$ & $1.8 \%$ & $5.3 \%$ & $16.0 \%$ \\
\hline & \multirow{2}{*}{\multicolumn{2}{|c|}{ Total }} & $\mathrm{N}$ & - & 53 & 208 & - & 69 & 66 & 210 & 606 \\
\hline & & & $\%$ del total & & $8.7 \%$ & $34.3 \%$ & & $11.4 \%$ & $10.9 \%$ & $34.7 \%$ & $100.0 \%$ \\
\hline
\end{tabular}

Se proveyeron instrucciones precisas y detalladas a los tutores y encargados para la elección aleatoria dentro de cada nivel educativo. Con estas instrucciones se aseguró la estandarización del procedimiento y que las muestras eran realmente aleatorias y no dependían de ningún criterio ad hoc momentáneo. 
Tabla 2. Comunalidades (cuestionario profesores)

\begin{tabular}{lll}
\hline & \multicolumn{2}{c}{ Componente } \\
\hline & Inicial & Extracción \\
\hline P_ITEM1 & 1.000 & .696 \\
\hline P_ITEM2 & 1.000 & .733 \\
\hline P_ITEM3 & 1.000 & .606 \\
\hline P_ITEM4 & 1.000 & .776 \\
\hline P_ITEM18 & 1.000 & .715 \\
\hline P_ITEM17 & 1.000 & .722 \\
\hline P_ITEM16 & 1.000 & .622 \\
\hline P_ITEM15 & 1.000 & .553 \\
\hline P_ITEM14 & 1.000 & .685 \\
\hline P_ITEM13 & 1.000 & .498 \\
\hline P_ITEM12 & 1.000 & .646 \\
\hline P_ITEM11 & 1.000 & .722 \\
\hline P_ITEM10 & 1.000 & .681 \\
\hline P_ITEM9 & 1.000 & .711 \\
\hline P_ITEM8 & 1.000 & .703 \\
\hline P_ITEM6 & 1.000 & .737 \\
\hline P_ITEM7 & 1.000 & .599 \\
\hline P_ITEM5 & 1.000 & .766 \\
\hline Método de extracción: Análisis de & Componentes \\
principales & & \\
\hline
\end{tabular}

Tabla 3. Matriz de componentes (a) (cuestionario profesores)

\begin{tabular}{lll}
\hline & \multicolumn{2}{c}{ Componente } \\
\hline & 1 & 2 \\
\hline P_ITEM1 & $\mathbf{. 8 1 4}$ & .182 \\
\hline P_ITEM2 & $\mathbf{. 8 1 2}$ & .273 \\
\hline P_ITEM3 & $\mathbf{. 7 0 0}$ & .341 \\
\hline P_ITEM4 & $\mathbf{. 8 6 6}$ & .161 \\
\hline P_ITEM18 & .262 & $\mathbf{. 8 0 4}$ \\
\hline P_ITEM17 & .200 & $\mathbf{. 8 2 6}$ \\
\hline P_ITEM16 & .103 & $\mathbf{. 7 8 2}$ \\
\hline P_ITEM15 & .141 & $\mathbf{. 7 3 0}$ \\
\hline P_ITEM14 & .208 & $\mathbf{. 8 0 1}$ \\
\hline P_ITEM13 & .379 & $\mathbf{. 5 9 5}$ \\
\hline P_ITEM12 & .334 & $\mathbf{. 7 3 1}$ \\
\hline P_ITEM11 & .317 & $\mathbf{. 7 8 8}$ \\
\hline P_ITEM10 & .298 & $\mathbf{. 7 6 9}$ \\
\hline P_ITEM9 & $\mathbf{. 8 1 7}$ & .208 \\
\hline P_ITEM8 & $\mathbf{. 7 7 9}$ & .311 \\
\hline P_ITEM6 & $\mathbf{. 8 2 8}$ & .227 \\
\hline P_ITEM7 & $\mathbf{. 6 8 5}$ & .360 \\
\hline P_ITEM5 & $\mathbf{. 8 5 6}$ & .183 \\
\hline
\end{tabular}

La tasa de prevalencia de TDAH se determinó a partir de una puntuación de corte (el percentil 95) para todas las escalas (inatención, hiperactividad/impulsividad, y combinado) y también para los dos tipos de informantes (padres y profesores, ver Anexo I). Los baremos utilizados para clasificar a los sujetos se obtuvieron previamente de la muestra de 2.395 alumnos que se habían seleccionado al azar. Por tanto, se consideró que un alumno presenta sospecha de TDAH inatento cuando tanto para padres como para profesores se igualaba o se superaba el percentil 95 sólo en la escala de inatención. En el caso de la hiperactividad/impulsividad se consideró sospecha de TDAH hiperactivo si se igualaba o superaba el percentil 95 en ambos informantes en la escala de hiperactividad. Y, finalmente, un alumno se consideró con sospecha de TDAH combinado cuando se igualaba o superaba el percentil 95 en ambos informantes en la escala total.

\section{RESULTADOS}

Mediante el análisis de componentes principales, analizamos la estructura interna de la escala del DSM-IV para ambos informantes (profesores vs. padres). Los resultados nos muestran una estructura factorial formada por dos factores principales o componentes que en su conjunto explican el $67.6 \%$ de la varianza total para los profesores, y un $51.2 \%$ para los padres. En las tablas 2 y 3 se presenta la matriz factorial 
rotada según el método varimax para la escala de profesores en la que aparece el componente extraído y la saturación de cada una de las funciones evaluadas de la escala.

Las tablas 4 y 5 recogen la matriz factorial rotada según el método varimax para la escala de padres.

En ambos casos, a la hora de determinar si los pesos factoriales son significativos se suele considerar que estos valores sean mayores de .30, y muy significativos si se aproximan a .50 (Comrey, 1985). Conforme a estos criterios podemos observar que existen dos factores o componentes, tanto en el caso de los profesores como de los padres.

Tabla 4. Comunalidades (cuestionario padres)

\begin{tabular}{|c|c|c|}
\hline & \multicolumn{2}{|c|}{ dimension1 } \\
\hline & Inicial & Extracción \\
\hline FA_ITEM1 & 1.000 & .558 \\
\hline FA_ITEM2 & 1.000 & .587 \\
\hline FA_ITEM3 & 1.000 & .477 \\
\hline FA_ITEM4 & 1.000 & .616 \\
\hline FA_ITEM16 & 1.000 & .436 \\
\hline FA_ITEM18 & 1.000 & .483 \\
\hline FA_ITEM17 & 1.000 & .508 \\
\hline FA_ITEM5 & 1.000 & .609 \\
\hline FA_ITEM15 & 1.000 & .507 \\
\hline FA_ITEM14 & 1.000 & .533 \\
\hline FA_ITEM13 & 1.000 & .278 \\
\hline FA_ITEM12 & 1.000 & .526 \\
\hline FA_ITEM11 & 1.000 & .582 \\
\hline FA_ITEM10 & 1.000 & .555 \\
\hline FA_ITEM9 & 1.000 & .520 \\
\hline FA_ITEM8 & 1.000 & .560 \\
\hline FA_ITEM7 & 1.000 & .328 \\
\hline FA_ITEM6 & 1.000 & .571 \\
\hline $\begin{array}{l}\text { Método de } \\
\text { principales. }\end{array}$ & Análisis & Compor \\
\hline
\end{tabular}

Tabla 5. Matriz de componentes (a) (cuestionario padres)

\begin{tabular}{|c|c|c|c|}
\hline & & \multicolumn{2}{|c|}{$\begin{array}{c}\text { Componente } \\
\text { dimension1 } \\
\end{array}$} \\
\hline & & 1 & 2 \\
\hline \multirow[t]{18}{*}{ dimension0 } & FA_ITEM1 & .728 & .168 \\
\hline & FA_ITEM2 & .741 & .193 \\
\hline & FA_ITEM3 & .604 & .335 \\
\hline & FA_ITEM4 & .768 & .160 \\
\hline & FA_ITEM16 & .069 & .657 \\
\hline & FA_ITEM18 & .229 & .656 \\
\hline & FA_ITEM17 & .199 & .684 \\
\hline & FA_ITEM5 & .774 & .102 \\
\hline & FA_ITEM15 & .040 & .711 \\
\hline & FA_ITEM14 & .178 & .708 \\
\hline & FA_ITEM13 & .345 & .399 \\
\hline & FA_ITEM12 & .330 & .646 \\
\hline & FA_ITEM11 & .317 & .694 \\
\hline & FA_ITEM10 & .284 & .689 \\
\hline & FA_ITEM9 & .701 & .168 \\
\hline & FA_ITEM8 & .680 & .313 \\
\hline & FA_ITEM7 & .514 & .252 \\
\hline & FA_ITEM6 & .737 & .166 \\
\hline \multicolumn{4}{|c|}{$\begin{array}{l}\text { Método de extracción: Análisis de componentes principales. } \\
\text { Método de rotación: Normalización Varimax con Kaiser. }\end{array}$} \\
\hline
\end{tabular}

En ambos informantes el primer componente extraído recoge los ítems que hacen referencia a la inatención, y el segundo componente aquellos que hacen referencia a la hiperactividad/impulsividad. Las estructuras factoriales (matrices de coeficientes estructura) de los padres y de los profesores presentan un patrón idéntico con los mismos valores altos en cada ítem de cada factor. En el caso de los profesores los ítems que presentan mayor saturación en el primer componente se refieren a "dificultades en seguir 
instrucciones", "dificultades para organizar tareas" y "evitar dedicarse a tareas que requieren esfuerzo mental sostenido", y, en el segundo componente, los ítems se refieren a "dificultades para esperar su turno", "interrumpir o interferir en las actividades de otros", y "estar siempre con prisas o actuar como si estuviera activado por un motor". En el caso de los padres, los ítems que presentan mayor saturación en el primer componente se refieren a "dificultades en seguir instrucciones", "dificultades en mantener la atención en tareas o actividades de juego", y "evitar dedicarse a tareas que requieren esfuerzo mental sostenido". Y, respecto al segundo componente, los ítems se refieren a "hablar en exceso", "estar siempre con prisas o actuar como si estuviera activado por un motor" y "mover en exceso manos y pies o removerse en su asiento".

Con el fin de conocer la relación existente entre los informantes para cada una de estos componentes que recoge el DSM-IV, se calculó la matriz de correlaciones. La tabla 6 recoge esta matriz, donde se puede observar que todas las correlaciones han resultado ser significativas. Estas correlaciones se han calculado a partir de las puntuaciones directas obtenidas en las distintas escalas. Se observa una validez convergente moderada entre las escalas de profesores y padres en el componente de inatención, donde se alcanza una correlación de .58, y en la escala total de .54, en cambio en el componente de hiperactividad/impulsividad se obtiene un coeficiente de .43. En el caso de la validez discriminante de las escalas inatención e hiperactividad/impulsividad se correlacionan 0.56 en los profesores, y 0.55 en los padres. En el caso de las correlaciones cruzadas entre inatención en profesores e hiperactividad/impulsividad en padres se obtiene un coeficiente de $0.43 \mathrm{y}$ en el caso de la inatención padres e hiperactividad/impulsividad en profesores de 0.31 .

Tabla 6. Matriz de correlaciones multifuente-multirrasgo

\begin{tabular}{|c|c|c|c|c|c|c|c|}
\hline & & $\begin{array}{c}\text { Inatención/pa } \\
\text { dres }\end{array}$ & $\begin{array}{l}\text { Hiperactividad- } \\
\text { impulsividad/ } \\
\text { padres }\end{array}$ & $\begin{array}{l}\text { Inatención/ } \\
\text { maestros }\end{array}$ & $\begin{array}{l}\text { Hiperactividad- } \\
\text { impulsividad/ } \\
\text { maestros }\end{array}$ & $\begin{array}{c}\text { Total/ } \\
\text { maestros }\end{array}$ & $\begin{array}{l}\text { Total/ } \\
\text { padres }\end{array}$ \\
\hline \multirow{3}{*}{ Inatento/padres } & Correlación de Pearson & 1 & $.558(* *)$ & $.581(* *)$ & $.319(* *)$ & $.524(* *)$ & $.888(* *)$ \\
\hline & Sig. (bilateral) & & .000 & .000 & .000 & .000 & .000 \\
\hline & $\mathrm{N}$ & & 2178 & 2106 & 2105 & 2106 & 2199 \\
\hline \multirow{3}{*}{$\begin{array}{l}\text { Hiperactividad- } \\
\text { impulsividad/ } \\
\text { padres }\end{array}$} & Correlación de Pearson & & 1 & $.346(* *)$ & $.437(* *)$ & $.436(* *)$ & $.877(* *)$ \\
\hline & Sig. (bilateral) & & & .000 & .000 & .000 & .000 \\
\hline & $\mathrm{N}$ & & & 2086 & 2085 & 2086 & 2178 \\
\hline \multirow{3}{*}{ Inatento/maestros } & Correlación de Pearson & & & 1 & $.566(* *)$ & $.911(* *)$ & $.525(* *)$ \\
\hline & Sig. (bilateral) & & & & .000 & .000 & .000 \\
\hline & $\mathrm{N}$ & & & & 2290 & 2291 & 2106 \\
\hline \multirow{3}{*}{$\begin{array}{l}\text { Hiperactividad- } \\
\text { impulsividad/ } \\
\text { maestros }\end{array}$} & Correlación de Pearson & & & & 1 & $.855(* *)$ & $.425(* *)$ \\
\hline & Sig. (bilateral) & & & & & .000 & .000 \\
\hline & $\mathrm{N}$ & & & & & 2290 & 2105 \\
\hline \multirow{3}{*}{ Total/maestros } & Correlación de Pearson & & & & & 1 & $.542(* *)$ \\
\hline & Sig. (bilateral) & & & & & & .000 \\
\hline & $\mathrm{N}$ & & & & & & 2106 \\
\hline \multirow{3}{*}{ Total/padres } & Correlación de Pearson & & & & & & 1 \\
\hline & Sig. (bilateral) & & & & & & \\
\hline & $\mathrm{N}$ & & & & & & \\
\hline
\end{tabular}


Se encontró una tasa global de prevalencia del $4.9 \%$, de los cuales el $3.1 \%$ fueron del subtipo inatento, el $1.1 \%$ hiperactivo, y el $0.7 \%$ combinado. En la tabla 7 presentamos los datos de prevalencia de TDAH según subtipos y sexo. La distribución de los subtipos no ha sido igual para ambos sexos $\chi 2_{(2)}=6.80, p<.05$. Esto significa que la tendencia no ha resultado igual en ambos sexos. El subtipo predominante ha sido el inatento $(64 \%)$, registrándose un mayor porcentaje en varones $(44.9 \%)$ que en mujeres (18.6\%). Le sigue el subtipo hiperactivo (22.9\%), también registrándose mayor porcentaje en varones $(18.6 \%)$ que en mujeres $(4.2 \%)$. Y, finalmente, el combinado $(13.6 \%)$ donde sólo se registraron varones.

Tabla 7. Tasas de sospecha de trastorno por déficit de atención e hiperactividad (TDAH) por subtipos y sexo

\begin{tabular}{|c|c|c|c|c|c|c|}
\hline & & & & btipo TDAH (percentil 95) & & Total \\
\hline & & & Inatento & Hiperactivo/impulsivo & Combinado & Inatento \\
\hline \multirow{4}{*}{ SEXO } & \multirow{2}{*}{ HOMBRE } & $\mathrm{N}$ & 53 & 22 & 16 & 91 \\
\hline & & $\%$ del total & $58 \%$ & $24 \%$ & $18 \%$ & $77.1 \%$ \\
\hline & \multirow{2}{*}{ MUJER } & $\mathrm{N}$ & 22 & 5 & 0 & 27 \\
\hline & & $\%$ del total & $81 \%$ & $19 \%$ & $.0 \%$ & $22.9 \%$ \\
\hline \multirow{2}{*}{ Total } & & $\mathrm{N}$ & 75 & 27 & 16 & 118 \\
\hline & & $\%$ del total & $64 \%$ & $23 \%$ & $14 \%$ & $100.0 \%$ \\
\hline
\end{tabular}

En la tabla 8 se presenta la tasa de prevalencia de TDAH por Islas. En este caso no se encontraron diferencias significativas en la distribución de los subtipos en función de la isla $\chi 2_{(12)}=5.77, p=.92$.

Tabla 8. Tasas de sospecha de trastorno por déficit de atención e hiperactividad (TDAH)

\begin{tabular}{|c|c|c|c|c|c|}
\hline \multirow{2}{*}{ ISLA } & \multicolumn{4}{|c|}{ Subtipo TDAH (percentil 95) } & \multirow{2}{*}{ Total } \\
\hline & & Inatento & Hiperactivo & Combinado & \\
\hline \multirow{2}{*}{ EL HIERRO } & $\mathrm{N}$ & 2 & 0 & 0 & 2 \\
\hline & $\%$ del total & $100 \%$ & $.0 \%$ & $.0 \%$ & $1.7 \%$ \\
\hline \multirow{2}{*}{ FUERTEVENTURA } & $\mathrm{N}$ & 3 & 0 & 0 & 3 \\
\hline & $\%$ del total & $100 \%$ & $.0 \%$ & $.0 \%$ & $2.5 \%$ \\
\hline \multirow{2}{*}{ GRAN CANARIA } & $\mathrm{N}$ & 31 & 14 & 8 & 53 \\
\hline & $\%$ del total & $58 \%$ & $26 \%$ & $16 \%$ & $44.9 \%$ \\
\hline \multirow{2}{*}{ LA GOMERA } & $\mathrm{N}$ & 2 & 1 & 1 & 4 \\
\hline & $\%$ del total & $50 \%$ & $25 \%$ & $25 \%$ & $3.4 \%$ \\
\hline \multirow{2}{*}{ LA PALMA } & $\mathrm{N}$ & 11 & 4 & 1 & 16 \\
\hline & $\%$ del total & $69 \%$ & $25 \%$ & $6 \%$ & $13.6 \%$ \\
\hline \multirow{2}{*}{ LANZAROTE } & $\mathrm{N}$ & 3 & 1 & 0 & 4 \\
\hline & $\%$ del total & $75 \%$ & $25 \%$ & $0 \%$ & $3.4 \%$ \\
\hline \multirow{2}{*}{ TENERIFE } & $\mathrm{N}$ & 23 & 7 & 6 & 36 \\
\hline & $\%$ del total & $64 \%$ & $16 \%$ & $17 \%$ & $30.5 \%$ \\
\hline \multirow{2}{*}{ Total } & $\mathrm{N}$ & 75 & 27 & 16 & 118 \\
\hline & $\%$ del total & $63 \%$ & $23 \%$ & $14 \%$ & $100.0 \%$ \\
\hline
\end{tabular}

Tampoco se encontraron diferencias significativas en la distribución de subtipos de TDAH en función del tipo de colegio (público vs. concertado) $\chi 2_{(2)}=5.86$, $p=.053$ tal y como se muestra en la tabla 9 . 
Por último, en el análisis de la relación entre subtipos TDAH y curso, tampoco se encontró una relación significativa $\chi^{2}(10)=7.39, p=.68$, y las tasas de prevalencia se recogen en la tabla 10.

Tabla 9. Tasas de sospecha de trastorno por déficit de atención e hiperactividad (TDAH)

\begin{tabular}{|c|c|c|c|c|c|}
\hline \multirow[b]{2}{*}{ TIPO DE COLEGIO } & \multicolumn{4}{|c|}{ Subtipo TDAH (percentil 95) } & \multirow[b]{2}{*}{ Total } \\
\hline & & Inatento & $\begin{array}{l}\text { Hiperactivo/ } \\
\text { impulsivo }\end{array}$ & Combinado & \\
\hline \multirow{2}{*}{ PUBLICO } & $\mathrm{N}$ & 54 & 21 & 16 & 91 \\
\hline & $\%$ del total & $59 \%$ & $23 \%$ & $18 \%$ & $77.1 \%$ \\
\hline \multirow{2}{*}{ CONCERTADO } & $\mathrm{N}$ & 21 & 6 & 0 & 27 \\
\hline & $\%$ del total & $78 \%$ & $22 \%$ & $.0 \%$ & $22.9 \%$ \\
\hline \multirow{2}{*}{ Total } & $\mathrm{N}$ & 75 & 27 & 16 & 118 \\
\hline & $\%$ del total & $64 \%$ & $23 \%$ & $13 \%$ & $100.0 \%$ \\
\hline
\end{tabular}

Tabla 10. Tasas de sospecha de trastorno por déficit de atención e hiperactividad (TDAH) por subtipos y curso

\begin{tabular}{|c|c|c|c|c|c|}
\hline \multirow[b]{2}{*}{ Curso } & & \multicolumn{3}{|c|}{ Subtipo TDAH (percentil 95) } & \multirow{2}{*}{$\begin{array}{c}\text { Total } \\
\text { Inatento }\end{array}$} \\
\hline & & Inatento & $\begin{array}{l}\text { Hiperactivo/ } \\
\text { impulsivo }\end{array}$ & Combinado & \\
\hline \multirow{2}{*}{ Primero } & $\mathrm{N}$ & 13 & 9 & 2 & 24 \\
\hline & $\%$ del total & $54 \%$ & $38 \%$ & $8 \%$ & $20.3 \%$ \\
\hline \multirow{2}{*}{ Segundo } & $\mathrm{N}$ & 18 & 5 & 4 & 27 \\
\hline & $\%$ del total & $67 \%$ & $18 \%$ & $15 \%$ & $22.9 \%$ \\
\hline \multirow{2}{*}{ Tercero } & $\mathrm{N}$ & 13 & 6 & 3 & 22 \\
\hline & $\%$ del total & $59 \%$ & $27 \%$ & $14 \%$ & $18.6 \%$ \\
\hline \multirow{2}{*}{ Cuarto } & $\mathrm{N}$ & 16 & 5 & 3 & 24 \\
\hline & $\%$ del total & $67 \%$ & $21 \%$ & $12 \%$ & $20.3 \%$ \\
\hline \multirow{2}{*}{ Quinto } & $\mathrm{N}$ & 7 & 1 & 3 & 11 \\
\hline & $\%$ del total & $64 \%$ & $9 \%$ & $27 \%$ & $9.3 \%$ \\
\hline \multirow{2}{*}{ Sexto } & $\mathrm{N}$ & 8 & 1 & 1 & 10 \\
\hline & $\%$ del total & $80 \%$ & $10 \%$ & $10 \%$ & $8.5 \%$ \\
\hline \multirow{2}{*}{ Total } & $\mathrm{N}$ & 75 & 27 & 16 & 118 \\
\hline & $\%$ del total & $63 \%$ & $23 \%$ & $14 \%$ & $100.0 \%$ \\
\hline
\end{tabular}

\section{DISCUSIÓN}

El principal objetivo de este estudio ha sido estimar la tasa de prevalencia del TDAH en la Comunidad Autónoma de Canarias a través de una muestra representativa de la población escolar, de edades comprendidas entre 6 y 12 años. Asimismo, se ha pretendido analizar si la distribución de los diferentes subtipos TDAH es diferente según el sexo, isla, tipo de colegio y curso. A diferencia de otros estudios que han utilizado en nuestro país baremos estadounidenses de origen para la clasificación de los sujetos (Cardo et al., 2007), en el presente estudio hemos utilizado puntuaciones normalizadas propias obtenidas de la población escolar canaria.

Previamente al análisis de la prevalencia se calcularon datos normalizados del DSM-IV para la población escolar canaria y, al mismo tiempo, se analizó la validez empírica del sistema de clasificación en subtipos. El DSM-IV distingue tres subtipos: combinado, predominio inatencional y predominio hiperactividad/impulsividad. En el análisis de componentes principales se pudo observar que en ambos informantes el 
primer componente extraído recoge los ítems que hacen referencia a la inatención, y el segundo componente aquellos que hacen referencia a la hiperactividad/impulsividad.

En líneas generales se ha encontrado una tasa de sospecha de TDAH del $4.9 \%$, siendo coincidente este resultado con estudios más recientes realizados en nuestro país, como el realizado en la isla de Mallorca (Cardo et al., 2007), que siguiendo una metodología muy similar a la empleada en esta investigación se encontró un $4.6 \%$ con un intervalo de confianza entre el 3 y el $6 \%$.

En cuanto a las diferencias por sexo la tendencia es claramente favorable a un predominio del trastorno en varones (Buitelaar y Van Engeland, 1996; Du Paul et al., 1998) aunque otros estudios no encontraron diferencias por sexo detectando incluso una tendencia por parte de las niñas a presentar tasas más elevadas (Cardo et al., 2007; Montiel-Nava et al., 2002). En nuestro caso, se encontraron diferencias significativas tal y como se había predicho. Esto es, la tendencia no ha resultado igual en ambos sexos, y se ha registrado un mayor porcentaje de varones identificados con sospecha de TDAH en comparación a las niñas. Se ha llegado a sugerir que el trastorno en casi todas sus manifestaciones, excepto el subtipo atencional, es más frecuente en niños que en niñas (Cardo y Severa, 2005), cosa que no ocurre en nuestro estudio.

Analizando los subtipos nos encontramos que el subtipo inatento es mucho más frecuente que el subtipo hiperactivo y que el combinado en la muestra detectada con sospecha de TDAH $(63.6 \%, 22.9 \%$ y $13.6 \%$, respectivamente). En cambio, la tendencia observada en población adolescente ha sido que el subtipo combinado es mucho más frecuente que el subtipo inatento y el subtipo hiperactivo $(52.2 \%, 34.8 \%$ y $13 \%$, respectivamente) (Rhode et al., 2000). No obstante, hay que tener en cuenta las limitaciones de los estudios que sólo utilizan diseño transversal para poder concluir de manera más consistente sobre la estabilidad de la prevalencia de subtipos con el paso del tiempo. En este sentido, en algunos estudios longitudinales se ha encontrado que la sintomatología motora del trastorno disminuye con la edad, mientras que la de tipo cognitivo-atencional tiende a mantenerse (Cardo y Servera, 2005).

Por último, no se han hallado diferencias significativas en la distribución de subtipos en las variables isla, tipo de centro y curso. Esto sugiere que el TDAH se ha mostrado un trastorno uniformemente repartido en función de estas variables en la población escolar canaria.

\section{Agradecimientos}

Esta investigación ha sido financiada por la Agencia Canaria de Investigación, Innovación y Sociedad de la Información de la Consejería de Educación, Universidades y Sostenibilidad del Gobierno de Canarias, con ref. PI 2007/012 del que es IP el primer autor. 


\section{REFERENCIAS}

Buitelaar, J.K. y Van Engeland, H. (1996). Epidemiological approaches. En S. Sandberg (Ed), Hyperactivity disorders of childhood (pp. 26-68). Cambridge: Cambridge University Press.

Cardo, E. y Servera, M. (2005). Prevalencia del trastorno de déficit de atención e hiperactividad. Revista de Neurología, 40, 11-15.

Cardo, E., Servera, M. y Llobera, J. (2007). Estimación de la prevalencia del trastorno por déficit de atención e hiperactividad en población normal de la isla de Mallorca. Revista de Neurología, 44, 10-14.

Comrey, A.L. (1985). Manual de Análisis Factorial. Madrid: Cátedra.

Cornejo, J.W., Osío, O., Sánchez, Y., Carrizosa, J., Sánchez, G., Grisales, H., Castillo-Parra, H. y Holguín, J. (2005). Prevalencia del trastorno por déficit de atención-hiperactividad en niños y adolescentes colombianos. Revista de Neurología, 40, 716-722.

Du Paul, G., Power, J.T., Anastopoulos, A.D. y Reid, R. (1998). ADHD-Rating Scales DSM-IV for parents and teachers. Nueva York: Guilford Press.

Herrera, G. (2005). Prevalencia del déficit atencional con hiperactividad en niños y niñas de 3 a 5 años de la ciudad de Chillán, Chile. Theoria, 14, 45-55.

Montiel-Nava, C., Peña., J.A., López, M., Salas, M., Zurga, J.R., Montiel-Barbero, I., Pirela, D. y Cardozo, J.J. (2002). Estimaciones de la prevalencia del trastorno por déficit de atenciónhiperactividad en niños marabinos. Revista de Neurología, 35, 1019-1024.

Montiel-Nava, C. Peña, J.A. y Montiel-Barbero, I. (2003). Datos epidemiológicos del trastorno por déficit de atención con hiperactividad en una muestra de niños marabinos. Revista de Neurología, 37, 815-819.

Pineda, D.A., Lopera, F., Henao, G.C., Palacio, J.D. y Castellanos, F.X. (2001). Confirmación de la alta prevalencia del trastorno por déficit de atención en una comunidad colombiana. Revista de Neurología, 32, 217-222.

Rhode, L.A., Biederman, J., Busnello, E.A., Zimmerman, H., Schmitz, M., Martins, S., et al. (2000). ADHD in a school sample of Brazilian adolescents. Journal of The American Academy of Child y Adolescent Psychiatry, 38, 716-722. 
ANEXO I

Attention-Deficit/Hiperactivity Disorder Rating Scales IV (ADHD RS-IV) para PROFESORES y PADRES o TUTORES LEGALES

\section{CUESTIONARIO PARA PROFESORES}

Fecha de Cumplimentación:

Datos del Centro:

\begin{tabular}{|l|l|}
\hline COLEGIO: & Público $\square \quad$ Concertado $\square$ \\
\hline ISLA: & Municipio: \\
\hline
\end{tabular}

Datos del alumno:

\begin{tabular}{|l|l|l|l|l|}
\hline CURSO: & GRUPO $^{1}:$ & $\mathrm{N}^{\circ}$ LISTA $^{2}:$ & $\begin{array}{l}\text { FECHA NACIMIENTO: } \\
\text { niño }\end{array}$ \\
niña
\end{tabular}

Por favor, señale con una " $X$ ” en la casilla correspondiente a sus respuestas:

\begin{tabular}{|c|c|c|c|}
\hline $\begin{array}{l}\text { P1. Presta atención insuficiente a los detalles y comete } \\
\text { errores por descuido en las tareas escolares o en otras } \\
\text { actividades }\end{array}$ & Nunca/raramente $\square$ & Algunas veces $\square$ & Con mucha frecuencia $\square$ \\
\hline $\begin{array}{l}\text { P2. Tiene dificultades para mantener la atención en tareas o en } \\
\text { actividades de juego. }\end{array}$ & Nunca/raramente $\square$ & Algunas veces $\square$ & Con mucha frecuencia $\square$ \\
\hline P3. Parece no escuchar cuando se le habla directamente & Nunca/raramente $\square$ & Algunas veces $\square$ & Con mucha frecuencia $\square$ \\
\hline $\begin{array}{l}\text { P4. Suele tener dificultades para seguir instrucciones y } \\
\text { finalizar tareas escolares, encargos u obligaciones. }\end{array}$ & Nunca/raramente $\square$ & Algunas veces $\square$ & Con mucha frecuencia $\square$ \\
\hline P5. Tiene dificultad para organizar tareas y actividades. & Nunca/raramente $\square$ & Algunas veces $\square$ & Con mucha frecuencia $\square$ \\
\hline $\begin{array}{l}\text { P6. Evita dedicarse a tareas que requieren un esfuerzo mental } \\
\text { sostenido (como trabajos escolares o domésticos) }\end{array}$ & Nunca/raramente $\square$ & Algunas veces $\square$ & Con mucha frecuencia $\square$ \\
\hline $\begin{array}{l}\text { P7. Pierde cosas que son necesarias para sus tareas o } \\
\text { actividades (por ejemplo: juguetes, ejercicios escolares, } \\
\text { lápices, libros o herramientas) }\end{array}$ & Nunca/ raramente $\square$ & Algunas veces $\square$ & Con mucha frecuencia $\square$ \\
\hline P8. Se distrae fácilmente & Nunca/raramente $\square$ & Algunas veces $\square$ & Con mucha frecuencia $\square$ \\
\hline P9. Es olvidadizo en las actividades diarias & Nunca/raramente $\square$ & Algunas veces $\square$ & Con mucha frecuencia $\square$ \\
\hline $\begin{array}{l}\text { P10. Mueve en exceso manos y pies, o se remueve en su } \\
\text { asiento }\end{array}$ & Nunca/raramente $\square$ & Algunas veces $\square$ & Con mucha frecuencia $\square$ \\
\hline $\begin{array}{l}\text { P11. Acostumbra a estar inquieto en su asiento en clase o en } \\
\text { otras situaciones en que se espera que permanezca sentado }\end{array}$ & Nunca/raramente $\square$ & Algunas veces $\square$ & Con mucha frecuencia $\square$ \\
\hline $\begin{array}{l}\text { P12. De forma reiterada corre o salta en situaciones en que es } \\
\text { inapropiado hacerlo }\end{array}$ & Nunca/raramente $\square$ & Algunas veces $\square$ & Con mucha frecuencia $\square$ \\
\hline $\begin{array}{l}\text { P13. Tiene dificultad para jugar o dedicarse tranquilamente a } \\
\text { actividades de ocio }\end{array}$ & Nunca/raramente $\square$ & Algunas veces $\square$ & Con mucha frecuencia $\square$ \\
\hline $\begin{array}{l}\text { P14. Está siempre con prisas o actúa como si estuviera } \\
\text { "activado por un motor" }\end{array}$ & Nunca/raramente $\square$ & Algunas veces $\square$ & Con mucha frecuencia $\square$ \\
\hline P15. Habla en exceso & Nunca/raramente $\square$ & Algunas veces $\square$ & Con mucha frecuencia $\square$ \\
\hline $\begin{array}{l}\text { P16. Suele dar las respuestas antes de haber sido completadas } \\
\text { las preguntas }\end{array}$ & Nunca/raramente $\square$ & Algunas veces $\square$ & Con mucha frecuencia $\square$ \\
\hline P17. Tiene dificultades para esperar su turno & Nunca/raramente $\square$ & Algunas veces $\square$ & Con mucha frecuencia $\square$ \\
\hline $\begin{array}{l}\text { P18. Interrumpe o interfiere en las actividades de otros ( por } \\
\text { ejemplo, en conversaciones o juegos) }\end{array}$ & Nunca/raramente $\square$ & Algunas veces $\square$ & Con mucha frecuencia $\square$ \\
\hline
\end{tabular}

¡Muchas gracias por su colaboración!

${ }^{1}$ Letra de identificación del grupo ("A", "B", "C",,..). Si sólo hay un grupo, deben introducir la letra "A"

${ }^{2} \mathrm{El}$ "No de lista" es el número de referencia del alumno/a y no puede variar a lo largo del desarrollo de la evaluación. 
CUESTIONARIO PARA

PADRE/MADRE/TUTOR O TUTORA LEGAL

Fecha de Cumplimentación:

Datos del Centro:

\begin{tabular}{|l|l|}
\hline COLEGIO: & Público $\square \quad$ Concertado $\square$ \\
\hline ISLA: & Municipio: \\
\hline
\end{tabular}

Datos del alumno:

\begin{tabular}{|c|c|c|c|c|}
\hline CURSO: & GRUPO $^{3}:$ & $\mathrm{N}^{\circ}$ LISTA $^{4}$ : & FECHA NACIMIENTO: & $\begin{array}{l}\text { GÉNERO: } \\
\text { niño } \square \text { niña } \square\end{array}$ \\
\hline
\end{tabular}

Por favor, señale con una " $X$ " en la casilla correspondiente a sus respuestas:

\begin{tabular}{|c|c|c|c|}
\hline $\begin{array}{l}\text { FA1. Presta atención insuficiente a los detalles y comete errores } \\
\text { por descuido en las tareas escolares o en otras actividades }\end{array}$ & Nunca/raramente $\square$ & Algunas veces $\square$ & Con mucha frecuencia $\square$ \\
\hline $\begin{array}{l}\text { FA2. Tiene dificultades para mantener la atención en tareas o en } \\
\text { actividades de juego }\end{array}$ & Nunca/raramente $\square$ & Algunas veces $\square$ & Con mucha frecuencia $\square$ \\
\hline FA3. Parece no escuchar cuando se le habla directamente & Nunca/raramente $\square$ & Algunas veces $\square$ & Con mucha frecuencia $\square$ \\
\hline $\begin{array}{l}\text { FA4. Suele tener dificultades para seguir instrucciones y finalizar } \\
\text { tareas escolares, encargos u obligaciones }\end{array}$ & Nunca/raramente $\square$ & Algunas veces $\square$ & Con mucha frecuencia $\square$ \\
\hline FA5. Tiene dificultad para organizar tareas y actividades & Nunca/raramente $\square$ & Algunas veces $\square$ & Con mucha frecuencia $\square$ \\
\hline $\begin{array}{l}\text { FA6. Evita dedicarse a tareas que requieren un esfuerzo mental } \\
\text { sostenido (como trabajos escolares o domésticos) }\end{array}$ & Nunca/raramente $\square$ & Algunas veces $\square$ & Con mucha frecuencia $\square$ \\
\hline $\begin{array}{l}\text { FA7. Pierde cosas que son necesarias para sus tareas o actividades } \\
\text { (por ejemplo: juguetes, ejercicios escolares, lápices, libros o } \\
\text { herramientas) }\end{array}$ & Nunca/ raramente $\square$ & Algunas veces $\square$ & Con mucha frecuencia $\square$ \\
\hline FA. Se distrae fácilmente & Nunca/raramente $\square$ & Algunas veces $\square$ & Con mucha frecuencia $\square$ \\
\hline FA. Es olvidadizo en las actividades diarias & Nunca/raramente $\square$ & Algunas veces $\square$ & Con mucha frecuencia $\square$ \\
\hline FA. Mueve en exceso manos y pies, o se remueve en su asiento & Nunca/raramente $\square$ & Algunas veces $\square$ & Con mucha frecuencia $\square$ \\
\hline $\begin{array}{l}\text { FA. Acostumbra a estar inquieto en su asiento en clase o en otras } \\
\text { situaciones en que se espera que permanezca sentado }\end{array}$ & Nunca/raramente $\square$ & Algunas veces $\square$ & Con mucha frecuencia $\square$ \\
\hline $\begin{array}{l}\text { FA. De forma reiterada corre o salta en situaciones en que es } \\
\text { inapropiado hacerlo }\end{array}$ & Nunca/raramente $\square$ & Algunas veces $\square$ & Con mucha frecuencia $\square$ \\
\hline $\begin{array}{l}\text { FA. Tiene dificultad para jugar o dedicarse tranquilamente a } \\
\text { actividades de ocio }\end{array}$ & Nunca/raramente $\square$ & Algunas veces $\square$ & Con mucha frecuencia $\square$ \\
\hline $\begin{array}{l}\text { FA. Está siempre con prisas o actúa como si estuviera "activado por } \\
\text { un motor" }\end{array}$ & Nunca/raramente $\square$ & Algunas veces $\square$ & Con mucha frecuencia $\square$ \\
\hline FA. Habla en exceso & Nunca/raramente $\square$ & Algunas veces $\square$ & Con mucha frecuencia $\square$ \\
\hline $\begin{array}{l}\text { FA. Suele dar las respuestas antes de haber sido completadas las } \\
\text { preguntas }\end{array}$ & Nunca/raramente $\square$ & Algunas veces $\square$ & Con mucha frecuencia $\square$ \\
\hline FA. Tiene dificultades para esperar su turno & Nunca/raramente $\square$ & Algunas veces $\square$ & Con mucha frecuencia $\square$ \\
\hline $\begin{array}{l}\text { FA. Interrumpe o interfiere en las actividades de otros (por ejemplo, } \\
\text { en conversaciones o juegos) }\end{array}$ & Nunca/raramente $\square$ & Algunas veces $\square$ & Con mucha frecuencia $\square$ \\
\hline
\end{tabular}

¡Muchas gracias por su colaboración!

Recibido: 30 de septiembre de 2011

Recepción Modificaciones: 20 de marzo de 2012

Aceptado: 22 de marzo de 2012

\footnotetext{
${ }^{3}$ Letra de identificación del grupo ("A", "B", “C", ...). Si sólo hay un grupo, deben introducir la letra "A"

${ }^{4} \mathrm{El}$ " $\mathrm{N}^{0}$ de lista" es el número de referencia del alumno/a y no puede variar a lo largo del desarrollo de la evaluación.
} 